\title{
Transformation to Dynamic Single Assignment Using a Simple Data Flow Analysis ${ }^{\star}$
}

\author{
Peter Vanbroekhoven ${ }^{1 \star \star}$, Gerda Janssens ${ }^{1}$, Maurice Bruynooghe ${ }^{1}$, and Francky \\ Catthoor $^{2}$ \\ 1 Katholieke Universiteit Leuven, Belgium \\ 2 Interuniversity MicroElectronics Center, Belgium
}

\begin{abstract}
This paper presents a novel method to construct a dynamic single assignment (DSA) form of array-intensive, pointer-free C programs (or in any other procedural language). A program in DSA form does not perform any destructive update of scalars and array elements, i.e., each element is written at most once. As DSA makes the dependencies between variable references explicit, it facilitates complex analyses and optimizations of programs. This makes it a preferred intermediate form for a number of compiler techniques of growing importance: parallelization, systolic array design, programming heterogeneous architectures, memory optimization, and verification of source code transformations.

Existing transformations into DSA perform a complex data flow analysis that is exponential in the program size and that only accepts input programs where all loop bounds, array indexation and conditionals are (possibly piecewise) affine expressions in the loop iterators. Our method removes irregularities from the data flow by adding copy assignments to the program, and then it can use simple data flow analyses.

The contributions of this paper are threefold. The DSA transformation presented scales very well with growing program sizes. It is quadratic in the program size -though our experiments indicate that it tends towards linearity- and is polynomial in the loop nest depth, while other existing methods have exponential complexity. Our DSA transformation overcomes a number of important limitations of existing methods; it allows any expression for loop bounds, indexation and conditionals, as long as constant bounds can be found for loop iterators. We have implemented the method and it is being used in the context of memory optimization and verification of those optimizations.
\end{abstract}

\section{Introduction}

In a program in dynamic single assignment (DSA) form, there is only one assignment at run time to each scalar variable or array element[7]. Essentially this form directly encodes all the information gathered by an array data flow analysis,

\footnotetext{
* Research supported by FWO Vlaanderen

** Supported by a specialization grant from the Institute for the Promotion of Innovation by Science and Technology in Flanders (IWT)
} 
much like static single assignment (SSA) directly encodes use-def chains in the program[6]. While SSA focuses on scalar variables, DSA is geared towards array variables that are analyzed element by element. An alternate form of DSA is a system of recurrence equations[9] (SRE). This form does not give an execution order to the different statements, and instead only specify the set of iteration points. SREs are usually used when the execution order of the original code does not matter, for example because a new execution order is determined anyway.

DSA form has essentially two advantages: analyses become simpler as each use can be linked to a single def by matching the array elements accessed, and there are more opportunities for reordering transformations. DSA form (and equivalently an SRE) is therefore used in a number of compiler techniques:

1. Parallelization The need for decreasing the restrictions on reordering transformations is necessary to get good results from parallelization[13, 7]. SREs are often used as a starting point as it reveals all inherent parallelism in a program. With the advent of multi-processor systems, multi-core processors, hyper-threading, multimedia extensions (AltiVec, MMX, SSE2), the demand for parallelism grows, especially within embedded systems. Also CPU pipelines supporting out-of-order execution benefit from more parallelism within a program.

2. Systolic arrays Procedural programs are transformed to SREs, which are then mapped onto systolic arrays, forming the basis for VLSI chip de$\operatorname{sign}[17]$.

3. Heterogeneous architectures Heterogeneous architectures are expected to become more and more common, especially in embedded systems[11]. These architectures can consist of multiple processors, dedicated chips, FPGAs, ... DSA form is used here to partition the code in a number of chunks that are distributed over the different components.

4. Memory optimization Optimization of data-intensive programs, like multimedia and network application, benefit from the use of DSA to simplify the transformations and to get a better result $[16,5]$.

5 . Verification of source code transformations Verification of source code transformations $[22,23]$ is shown to gain both simplicity and power by the use of DSA.

The contribution of this paper is a new method for transformation to DSA that offers two advantages over existing work:

1. It overcomes a number of limitations in existing methods. Our method is not limited to (possibly piecewise) affine expressions for loop bounds, conditionals and array indexation as are $[7,10]$, but instead deals with general expressions including data-dependent expressions which are prevalent even in simple programs like Gaussian elimination with pivoting. The sole restriction is that constant bounds must be found on the loop iterators.

2. It is quadratic in the program size and polynomial in the depth of the loop nests, while existing methods are exponential. 


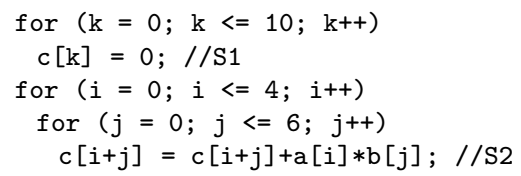

Fig. 1. Polynomial multiplication 1.

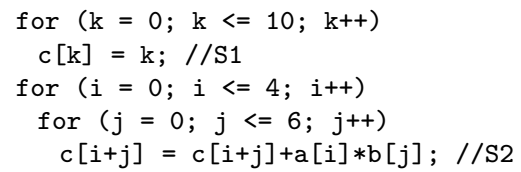

Fig. 2. Polynomial multiplication 2.

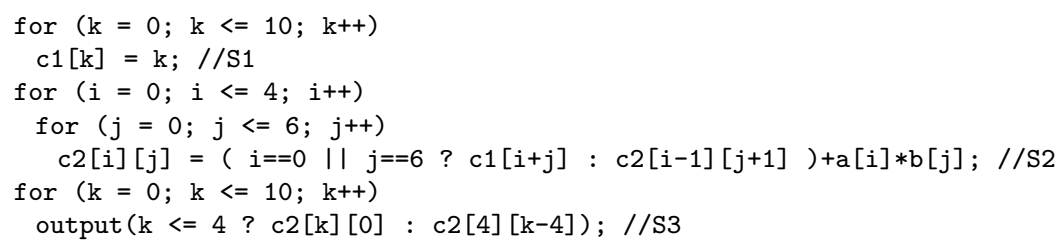

Fig. 3. Dynamic single assignment version of Fig. 2.

Moreover the implementation has been successfully used in the context of memory optimizations [5] and verification of those transformations [22, 23]. For more detailed results on the use with verification, see [21].

Section 2 discusses related work and situates our contribution. Section 3 introduces our approach. We explain the preparatory steps in Sec. 4, we do the DSA transformation in Sec. 5 and Sec. 6 discusses some additional steps. Sec. 7 rounds off the complexity analysis and Sec. 8 presents results of applying our prototype implementation to a number of multimedia kernels. Finally, Sec. 9 concludes.

\section{Related Work}

In optimizing compilers, data flow analyses are used to determine what optimizations are possible and how they should be done [1]. Forward data flow analyses propagate information from definitions of variables to their uses; they need to know which definitions reach which uses. One can distinguish the following queries (illustrated on the program in Fig. 1):

- What definitions reach a given use? The definitions S1 and S2 reach the use of $\mathrm{c}$ in statement $\mathrm{S} 2$.

- Under what condition does each definition reach a given use? For S2 there are two reaching definitions. The reader can verify that $\mathbf{S 1}$ reaches $\mathrm{S} 2$ when $i$ is 0 or when $j$ is 6 . S2 reaches itself in all other cases.

- What instance of a definition reaches what instance of a use? Statement S2 has an instance for each combination of values for $i$ and $j$, and the reaching definition varies with the instance of $\mathbf{S} 2$. The instance for $i=i_{1}$ and $\mathbf{j}=j_{1}$ is denoted $\mathbf{S} 2\left(i_{1}, j_{1}\right)$. For $\mathbf{S} 2\left(i_{1}, j_{1}\right)$ the reaching definition is $\mathbf{S} 1$ when $i_{1}=0$ or $j_{1}=6$. The instance of $\mathbf{S} 1$ that reaches $\mathbf{S} 2\left(i_{1}, j_{1}\right)$ is $\mathbf{S} 1\left(i_{1}+j_{1}\right)$.

The amount of information these three questions ask for increases, and so does the difficulty to answer them accurately. Classic compiler theory considers only 
the first question; this limits the amount of optimization, e.g., in Fig. 1, S1 assigns a constant 0 to $\mathrm{c}$, but constant propagation would not be done because $\mathrm{c}$ changes value in the second loop. The information asked for in the first question can be encoded efficiently in the program (possibly in the intermediate representation) by putting it in static single assignment (SSA) form [6]. In SSA form there is only one assignment to each variable in the program text, making the search for reaching definitions an exercise in variable name matching. A $\phi$-function is used to select the right reaching definition in case there is more than one.

The answer to the second question - if it can be answered for a particular case - can be regarded as the $\phi$-functions of SSA form that are made explicit. So in Fig. 1, instead of assuming that the $c[i+j]$ we read can be produced by either $\mathrm{S} 1$ or $\mathrm{S} 2$, we can deduce that it is produced by $\mathrm{S} 1$ when $i$ is 0 or $j$ is 6 . This knowledge allows us to split $\mathbf{S} 2$ on that condition and to do constant propagation to the part where the condition is true. The same technique is used to do copy propagation in [12], although it is not clear for which programs they can do this and how far they can go. Because it is typically useful for optimizing arrayintensive programs - where the savings in memory accesses usually outweigh the cost of splitting statements - the form that encodes the answer to the second question explicitly could be referred to as array SSA (ASSA).

Finally, the answer to the third question completes the information about reaching definitions by indicating the exact instance of a statement that reaches a given use, which is done by giving the values of the iterators of surrounding loops. This information is necessary for optimizations such as general expression propagation. Consider Fig. 2 where the initialization of c to zero is replaced by an expression depending on the iterator $\mathrm{k}$. If we want to propagate $\mathrm{S} 1$ to $\mathrm{S} 2$ again, we need to determine the correct value of $\mathrm{k}$ which will obviously depend on $i$ and $j$. The third question supplies us with exactly this information. This information can be represented explicitly by putting the program in dynamic single assignment (DSA) form as presented by Feautrier in [7].

A program in DSA form assigns each array element or scalar variable only once during execution. The DSA form of the program in Fig. 2 is shown in Fig. 3. We have added an extra statement at the end to indicate where the final coefficients of the product end up being stored. For the conditional split-up we use the $\mathrm{C}$ operator ?:. In statement S2, the condition selects between two reaching statements, while in statement S3, it selects between different expressions for the reaching instance. It is surprising how clear the answer to the questions above is from this program. For example S2 reads both $\mathrm{c} 1$ and c2, thus both S1 and S2 reach it. It reads $c 1$ when $i$ is 0 or $j$ is 6 , hence that is when $S 1$ reaches $S 2$. The exact element read by a given instance $\mathrm{S} 2\left(i_{2}, j_{2}\right)$ is $\mathrm{c} 1\left[i_{2}+j_{2}\right]$, so it is instance $\mathrm{S} 1\left(i_{2}+j_{2}\right)$ that reaches $i . e .$, for $\mathrm{k}$ equal to $\left(i_{2}+j_{2}\right)$. Despite the information being quite detailed, retrieving it is again simply a matter of matching.

Besides providing a compiler with very detailed data flow information, DSA form can enable more transformations. In DSA form, every value produced by an assignment is given its own memory location. Sharing memory locations limits the order in which values are put in those locations and are read from them, 
and thus limits optimizations that involve moving code or reordering execution. For example a value should not be put in a memory location that is occupied by another value that is still needed by the program, which is called a false dependency. The only constraints left after transformation to DSA are true dependencies which specify that the program should only read from a memory location after a value was written to it. As the name indicates, this kind of dependency is inherent to the program. In summary, DSA enables all reorderings of instructions as allowed by the true dependencies. So DSA is a stronger single assignment form intended for the computational kernels which require advanced transformations or optimizations, e.g., to cope with the ever growing demands of multimedia and network applications[16,5], or to map onto the hardware part of an embedded system.

In [7] an automated method is proposed to translate code consisting of assignments to arrays that are arbitrarily nested in for-loops to DSA form, with the limitation that all loop bounds, conditions and indexation are affine expressions of loop iterators and a number of parameters. These limitations are relaxed slightly in [10] by allowing certain kinds of modulo and integer division in expressions for loop bounds, indexation and conditions. Methods to translate to SREs exist as well, e.g., [4], but they lack the generality of [7] and [10]. However the approach of [7] can be adapted very easily to generate SREs, which is done by $[2]$.

Finally we note that besides the simple categorization of SSA - ASSA DSA, there are variations in between. For example [3] defines gated single assignment (GSA) which uses $\gamma$ functions -instead of $\phi$ functions- that have an extra selection predicate as parameter. Another example is weak DSA [14] which is constructed from ASSA form (which uses extra variables to reconstruct the original data flow), and which relaxes the condition of DSA by allowing multiple assignments to a single scalar or array element as long as the value assigned is the same. It is not stated in the report what the advantages of weak DSA are.

\section{Our Approach}

Few programs are written in DSA form. Languages that impose DSA form, like Silage [8] or Single Assignment C [18], exist but are not widely used because DSA form can be awkward to write. Most applications are written in a multiple assignment ${ }^{3}$ form. As DSA form is the ideal form to do exact array analyses and transformations on, such programs should be transformed to DSA form before applying optimizations. This is tedious and error-prone when done manually, hence automation is needed.

The methods in $[7,10]$ have limited applicability as they do not deal with programs that contain data dependent indexation or conditions, as is the case in e.g., Gaussian elimination or motion estimation. This is in our experience an important limitation. Another disadvantage of [7] is that it breaks down for larger

\footnotetext{
${ }^{3}$ As opposed to (dynamic) single assignment.
} 
programs. The limited scalability is a problem since global optimizations inline functions to optimize over function boundaries. Indeed, aggressive optimizations usually require specializing each call-site anyway to obtain the best results.

Our method realizes a scalable DSA transformation. In practice it is linear in the program size (for constant loop depth). Our method extends on existing methods; it handles all pointer-free code with static memory allocation. It is restricted to a single function though, but this is not a real limitation because functions are inlined as argued above.

The most important obstacle for obtaining a scalable, generally applicable DSA transformation is that the three questions outlined in Sect. 2 need to be answered exactly. The data flow analysis presented in [7] gives exact answers, but because of that it is not generally applicable and slow. Data flow analyses as presented in [1] give approximate answers when control flow paths join, or when an array is accessed, and because of that are generally applicable and fast. The observation underlying our approach is that we can do away with approximations without sacrificing speed when all variables are scalars, there are no if-statements, and every assignment is executed at least once in every iteration of every surrounding loop. In case the program does not have these properties, we apply simple transformations, basically adding copy statements, until it does.

The fact that our DSA transformation method adds many copy operations to the transformed code seems to imply that it is useless in the context of optimization - it both increases memory use and increases the number of memory accesses. This is however no problem for example for functional verification of global transformations $[20,23]$ which is very powerful but requires that programs be specified in DSA form. One of the transformations it is able to verify requires it to handle addition of copy operations, such that it can look right through the copy operations our method adds. This verification method can handle certain classes of data dependent conditions, but up to now there was no general way of transforming programs with data dependent conditions to DSA form. The presented work remedies that.

The DSA form we obtain can be made more usable by applying advanced copy propagation [24] on it. This removes the bulk of the added copy operations. Currently, advanced copy propagation deals with the same class of programs as the DSA method of [7]; we are extending it to handle the broader class of programs that our DSA method can transform.

Our running example is discrete convolution (Fig. 4(a)). The code assumes two signals are given. The first signal consists of 10 samples that are stored in array a. The second signal consists of 1000 samples that are stored in array b. The resulting 1009 samples are stored in array c. We focus on array c only from now on. Features of programs used in the complexity analysis along with the symbols used to denote them are shown in Fig. 4(b), whose right hand side column contains the corresponding values for our example program. 


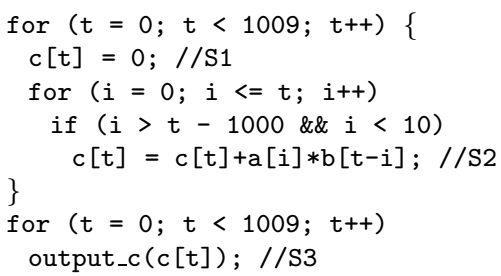

(a) Our running example.

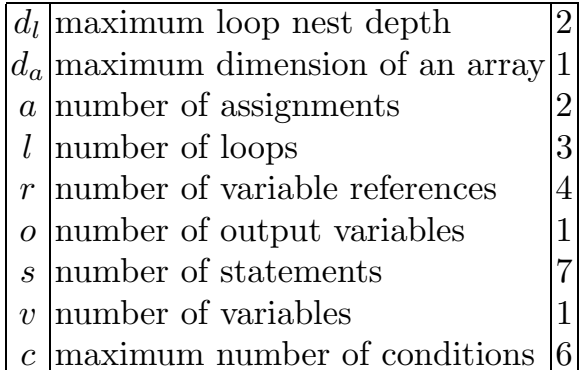

(b) Program characteristics.

Fig. 4.

The DSA transformation consists of several steps that are described in subsequent sections. The general overview of the DSA transformation is shown in Fig. 5, with references to the corresponding sections.

\section{Preparatory steps}

From array variables to scalars The main difference between scalars and arrays is that scalars are assigned as a whole while arrays are typically assigned element per element. One way to handle this, as suggested in [6], is to introduce an Update operation for each assignment to an array element that builds a whole new array with all elements equal to the corresponding elements of the old array except for one element that gets a new value. We use a slightly modified notation emphasizing that the array can be treated as a scalar. The result for our running example is shown in Fig. 6(a). The Ac functions are technically not necessary as they can be trivially replaced by direct array references, but they emphasize again that $\mathrm{c}$ is treated as a scalar. The meaning of an Up function is shown in Fig. 6(b).

Every Up function has the old array as parameter (here it is still the same as the new array), a number of indexation expressions equal to the number of array dimensions, and the right hand side of the assignment. Setting up these functions is straightforward, and is $O\left(d_{a}\right)$ with $d_{a}$ the maximum number of array dimensions in the program. For $a$ assignments, this takes $O\left(d_{a} \cdot a\right)$ time.

Execute every assignment in every iteration The aim is now to transform the program such that each assignment is executed at least once in each iteration of each surrounding loop. The two causes preventing the execution of an assignment are if-statements and loops that have zero iterations (possibly depending on the values of surrounding iterators). The simplest way to overcome these problems is to transform away if statements and replace all loop bounds 


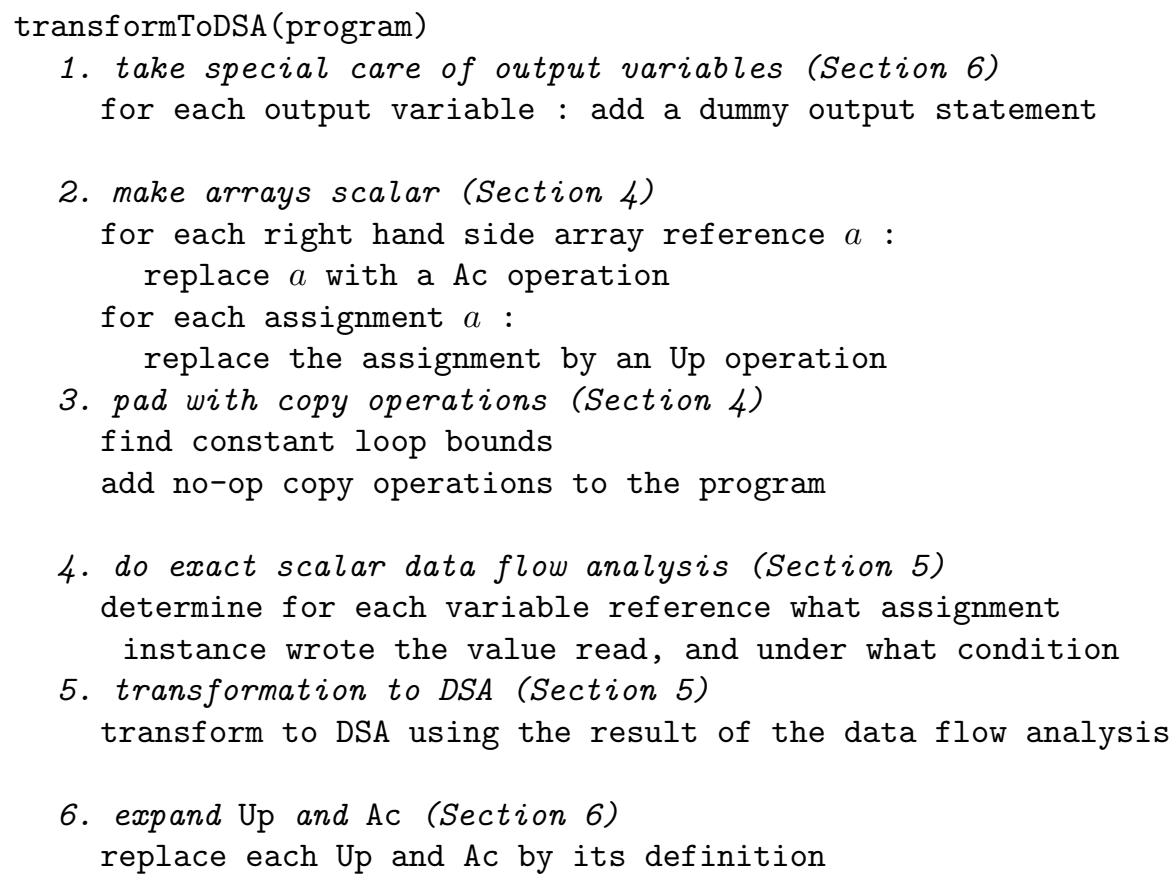

Fig. 5. High-level script of DSA transformation.

by constants. If these constant upper and lower bounds on iterator values indicate that the body of the loop is never executed, we remove it as the loop was useless to begin with.

With a quick inspection of the program in Fig. 6(a), we determine that the maximum value for the $i$ iterator is 9 . We can use this as upper bound on the $i$ loop provided we move the condition that $i$ should be smaller than $t$ to the if-statement. What remains now is the condition. The first step in removing the condition is to add an else-branch to each if-statement - if not yet present - and add a no-op assignment for each variable assigned in the other branch. For our running example this results in Fig. 7(a). Note that now c is always assigned a value, regardless of the value of the condition of the if-statement. So rather than selecting whether something is assigned, it selects what is assigned. This can be made explicit in $\mathrm{C}$ as shown in Fig. 7(b). Let us now again answer what the last statement is that assigned to c before arriving at S3, and for what value of the surrounding iterators. This has become trivial; the last assignment is done by $\mathrm{S} 2$ for $\mathrm{t}$ equal to 1008 and $\mathrm{i}$ equal to 9 .

When all bounds and conditions are affine functions of the iterators, we can use linear programming [19] to find the extremal values for the iterators. Linear programming is polynomial in the problem size, which can be measured by the number of loop bounds and conditions governing a statement. If we call the maximum number of these conditions $c$, then finding two bounds for each of the 


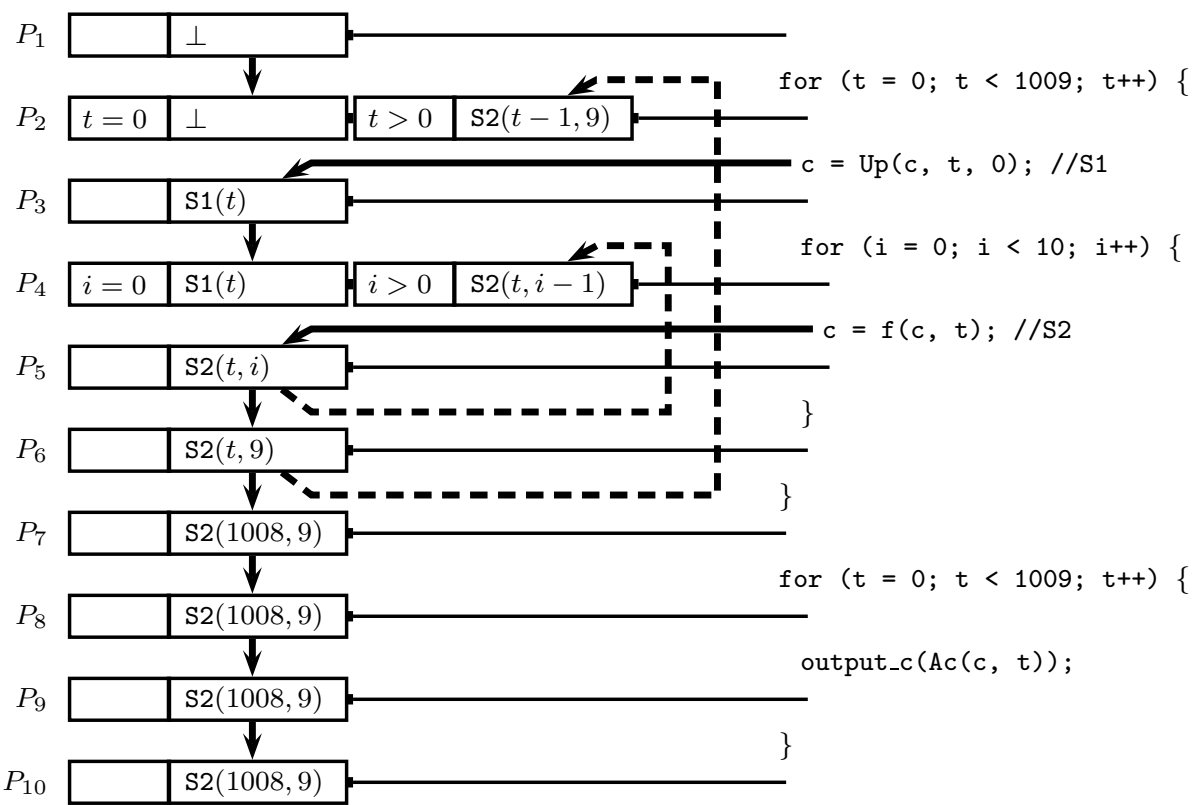

Fig. 8. Exact reaching definitions analysis on Fig. 7(b).

through $P_{10}$, one before and after each assignment, and one at the start and at end of each loop. At each program point we keep the reaching definition instances and the conditions under which they reach.

The number of reaching definitions to distinguish, and the conditions for each, are determined as follows:

- At the start of the program $\left(P_{1}\right)$, we distinguish one case and denote the reaching instance with $\perp$. This can be interpreted as either an uninitialized variable, or the initial value of the variable wherever it may come from.

- Upon entering a loop that contains an assignment to the variable we are analyzing (for example $P_{2}$ ), we split cases. Either we are in the first iteration of the loop $(t=0)$, in which case we distinguish the same reaching definitions as just before the loop (in the case of $P_{2}$ there is only one), or we are in a later iteration $(\mathrm{t}>0$ ), in which case the assignment in the loop has been executed at least once and has killed all reaching definitions from before the loop. In the latter case the reaching definition is taken from the end of the previous iteration of the loop. In case of $P_{2}$, the end of the loop is $P_{6}$, and for $\mathrm{t}>0$, the reaching definition there is found to be $\mathrm{S} 2(t, 9)$. Taking into account that we are in the next iteration of the $t$ loop, the reaching definition to $P_{2}$ becomes $\mathrm{S} 2(t-1,9)$.

- Upon entering a loop that contains no assignment to the variable being analyzed (e.g., $\left.P_{8}\right)$, the reaching definitions are obviously not affected and hence they are just copied from before the loop. 
- Just after a loop $\left(e . g ., P_{6}\right)$, the reaching definitions are those that reach the end of the last iteration of that loop. The reaching definitions to $P_{6}$ are those at $P_{5}$ for $i=9$. There is only one, and filling in $i=9$ gives $\mathbf{S} 2(t, 9)$.

- Directly after an assignment $\left(e . g ., P_{5}\right)$ all other reaching definitions are killed. Thus the assignment is the only reaching definition, and the reaching instance is obviously the one from the current iteration of the loop. For $P_{5}$ this is $\mathbf{S} 2(t, i)$.

The reader can easily verify that these rules unambiguously determine the cases to discern at each program point and where the reaching definition for each case comes from.

The maximum number of reaching definitions to discern at each program point is one more than the loop depth. At the start of the program we start with one case and zero loop depth. When going through a loop without an assignment of interest, nothing changes and the number of cases is smaller than the maximum. When entering a loop with an assignment of interest, one case is added (hence the maximum number of cases is at worst one more than the loop depth). After the assignment, the number of reaching definitions are reset to one, which is also the number of reaching definitions at the end of the loop. With $d_{l}$ the maximum loop depth, the amount of information kept at each program point is $O\left(d_{l}^{2}\right)$. Calculation of this information for all program points, $O(s)$ in number, and for all variables, $v$ in number, requires a total time $O\left(s \cdot d_{l}^{2} \cdot v\right)$. This is because all information can be calculated without any complex bookkeeping by just going over the program twice.

Transformation to DSA form In multiple assignment code involving only scalars, there are two causes of multiple assignments to the same variable; either there are two assignments to the same variable, or an assignment is in a loop. The former cause is removed by renaming the variables in the left hand side of each assignment such that they each write to a different variable. Typically this is done by adding a sequence number at the end of the variable. The latter cause is removed by changing the variable assigned to an array with one dimension for each surrounding loop, and we index that dimension with the iterator of the corresponding loop. For our running example this is shown in Fig. 9.

After adjusting the left hand sides, we still need to adjust the variable accesses in the right hand sides. It is possible that a number of assignments (definitions) reach a variable access, and which one needs to be read from depends on the values of the iterators. This information was derived by applying the reaching definitions analysis previously described.

For our example, $\mathrm{S} 1(t)$ is reached by $\perp$ when $t=0$. In other words when $\mathrm{S} 1$ is executed for $t$ equal to 0 , we should read from the variable written by the implicit assignment at the start of the program, which is just c. If $t>0, \mathrm{~S} 1(t)$ is reached by $\mathbf{S} 2(t-1,9)$. So when $\mathbf{S} 1$ is executed for $t$ larger than 0 , we should read from the variable written by $\mathrm{S} 2(\mathrm{t}-1,9)$, which is $\mathrm{c} 2$ [t-1] [9]. Because of the special form of the left hand sides, the correspondence between $\mathrm{S} 2(t-1,9)$ and $c 2[t-1]$ [9] is direct. For S2 and S3, the same direct correspondence is in 


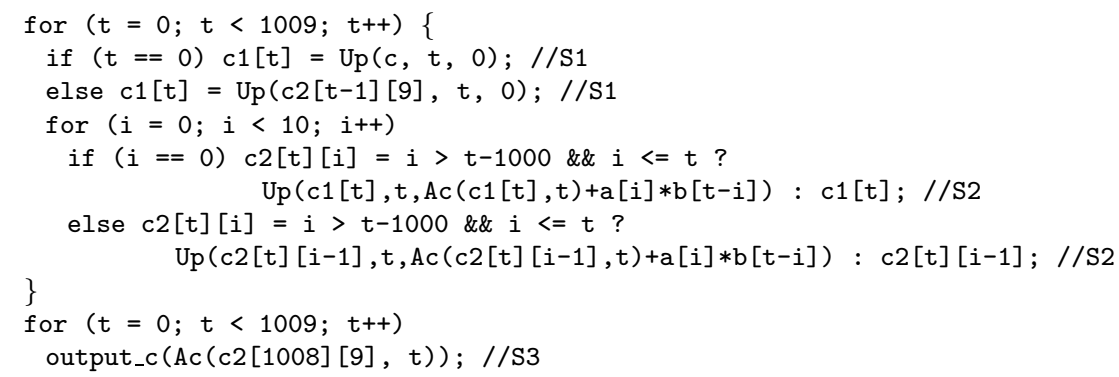

Fig. 9. Converting Fig. 7(b) to full DSA form.

order and the adjustment for the references to c in S2 and S3 are equally trivial. Filling everything in in Fig. 7(b) results in Fig. 9.

Adding the sequence number to the left hand side of assignment is a constant time operation, and because we need to add a number of dimensions equal to the number of surrounding for-loops, this step is $O\left(d_{l} \cdot a\right)$ with $d_{l}$ the maximum loop nest depth for each of the $a$ assignments. The adjustment of the right hand sides is executed for each variable reference, $r$ in number. This requires little more than just copying the expressions from the calculated reaching definitions, whose worst case size for each reference is $O\left(d_{l}^{2}\right)$. The complexity of this step is thus $O\left(r \cdot d_{l}^{2}+d_{l} \cdot a\right)$.

\section{Additional steps}

Expansion of Up and Ac Finally, we need to replace the Up and Ac function by their respective implementations. This is straightforward given the simple definition of these two functions.

There is an Up function for every assignment - at most $a$ in number - which on expansion gets an extra number of loops - one for each dimension of the original array such that the whole array can be written. The maximal dimension of the array reference is then $d_{l}+d_{a}$, and thus the size of the generated array references is $O\left(d_{l}+d_{a}\right)$. Since the generation is direct, the expansion of all Up functions requires a time that is $O\left(\left(d_{l}+d_{a}\right) \cdot a\right)$. There is an Ac function at every array reference in the original program - at most $r$ in number - which should be replaced with a new array reference with maximum dimension again $d_{l}+d_{a}$. The expansion of all Ac functions is then $O\left(\left(d_{l}+d_{a}\right) \cdot r\right)$. The total time needed for this step is $O\left(\left(d_{l}+d_{a}\right) \cdot(a+r)\right)$.

Output variables The piece of code we have been transforming to DSA calculates some result of interest to the user of that code, stores it in c, and at the end S3 outputs it using the output_c function. After transformation to DSA, this result is no longer stored in c. This is no problem as the method adjusts S3 as well to read from the newly added arrays - in this case c2. This is under the 


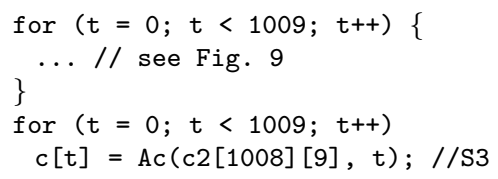

Fig. 10. Fig. 9 with output in c.

assumption that we are only interested in the numbers output by output_c, and not in the values stored in c at the end of the program. Usually only the computational kernels are transformed, and these kernels pass their results to the rest of the application through output arrays. In this case, our transformation needs to assure that the transformation does not change the values stored in these arrays after execution of the kernel. However, the code of Fig. 9 no longer stores any values in array c. We can trivially solve the problem by adding dummy output statements at the end of the program for each output array that output the complete arrays. In our running example we can consider S3 to be such a dummy output statement. The only thing left to do is to expand this dummy output statement such that it writes to c instead. This results in Fig. 10.

This step adds dummy output statements for $o$ output variables. The number of loops generated and the number of dimensions in the indexation are both equal to the dimension of the output variable, i.e., $d_{a}$. The generated statements have a total size of $O\left(o \cdot d_{a}\right)$, which is also the time complexity. Each of these dummy assignments has exactly one reaching definition, namely the preceding assignment to the same variable which can be determined in constant time, but finding the indexation of size $O\left(d_{l}\right)$ requires a time that is $O\left(d_{l}\right)$. Setting up the left hand side requires $O\left(d_{a}\right)$ operations, the right hand side requires $O\left(d_{l}+d_{a}\right)$ operations, giving $O\left(d_{l}+d_{a}\right)$ operations per output variable or $O\left(\left(d_{l}+d_{a}\right) \cdot o\right)$.

Extensions We have described our DSA transformation for the case where all loop bounds, indexation and conditions are affine functions of surrounding iterators. This fact is only used in Sec. 4 to find a constant upper and lower bound for the iterators. Subsequent steps work with those constants instead of the original loop bounds. The sole preceding step is the introduction of the Up and Ac functions, but they take along the indexation literally without analyzing it. This is illustrated in Fig. 6(b) where index is copied literally.

In general, conditions can be data dependent or non-affine, which prevents us from simply using linear programming to find the upper and lower bounds for the loops. We distinguish between bounds containing modulo operations and general non-affine bounds. When the bounds do contain modulo, we can translate the problem of finding a constant upper and lower bound to an equivalent problem that is affine. It is well known that modulo and integer division can be modeled using existential variables, e.g., saying that $a=b \bmod c$ is the same as saying $\exists q \in \mathbb{Z}: 0 \leq b-q \cdot c<c \wedge a=b-q \cdot c$. For the purpose of finding bounds, we can drop the requirement that $q$ should be integer. The condition has become linear and $q$ is just an extra variable in the linear programming problem. 
For general non-affine expressions, including data dependent ones, we can take several approaches:

- Find the extremal values for the iterators in the iteration domain with all expressions that are not affine functions of the iterators discarded. This does not work when the only expression bounding an iterator is not affine, but it was applicable in most experiments we have performed.

- Use the range of the data type of the iterator as upper and lower bound. This is a crude approximation, but it does not affect our intended use. For both verification and optimizations like advanced copy propagation, the iteration domains would become larger measured by the number of points in it, but their representation stays the same size - only a constant needs to be changed.

- A more advanced technique analyzes the range of values that data can take and uses this to find a tighter bound for data dependent conditions. This analysis is actually easier on DSA programs and should be performed after the DSA transformation to tighten the bounds. This is outside the scope of this paper.

With the above extensions we can conclude that our approach works for general kinds of conditions, loop bounds and indexation.

\section{Complexity analysis}

Since the whole DSA transformation method is the succession of each of the separate steps, the total complexity is simply the sum of the complexities of all steps. This results in a grand total of $O\left(d_{a} \cdot a+(p(c) \cdot l+a)+s \cdot d_{l}^{2} \cdot v+d_{l} \cdot a+r \cdot d_{l}^{2}+\right.$ $\left.\left(d_{l}+d_{a}\right) \cdot(a+r)+\left(d_{l}+d_{a}\right) \cdot o\right)=O\left(d_{a} \cdot(a+r+o)+d_{l} \cdot\left(s \cdot d_{l} \cdot v+r \cdot d_{l}+a+o\right)+p(c) \cdot l+a\right)$. Assuming that loop depth and array dimension are bounded by a constant, this simplifies to $O(o+r+a+s \cdot v+p(c) \cdot l)$. Because every measure in this formula is worst case proportional to the size of the program, our method is polynomial in the size of the program. $c$ is typically proportional to the depth of nesting, hence we can reasonably assume that $p(c)$ is bounded by a constant as well, and we obtain $O(o+a+s \cdot v+r+l)$. The presence of $s \cdot v$ in this expression is due to the reaching definitions analysis, and this is the only term that could have a tendency to grow large because as the number of statements $s$ increases, the number of variables $v$ is likely to increase as well. This makes our method quadratic. However, our experiments suggest that our method tends towards linearity. The difference between our experiments and our analysis is probably caused by the fact that the big- $O$ notation discards the constant coefficients (when some complexity is $O(2 \cdot n)$, it is also $O(n)$ and vice versa). This seems to indicate that the coefficients of the linear parts of the complexity weigh heaviest - most notably $p(c)$ can be quite a large constant. 


\begin{tabular}{|l|c|l|l|c|}
\hline benchmark & $\mathrm{LOC}$ & $d_{l}$ & time & Feaut. \\
\hline gauss 1 & 16 & 2 & $0.024 \mathrm{~s}$ & $\mathrm{X}$ \\
\hline gauss 2 & 17 & 2 & $0.027 \mathrm{~s}$ & $\mathrm{X}$ \\
\hline gauss_dd 1 & 65 & 3 & $0.331 \mathrm{~s}$ & \\
\hline gauss_dd 2 & 64 & 3 & $0.284 \mathrm{~s}$ & \\
\hline mp3_1 & 75 & 4 & $4.946 \mathrm{~s}$ & \\
\hline mp3_2 & 66 & 4 & $1.898 \mathrm{~s}$ & \\
\hline qsdpcm & 495 & 12 & $41.950 \mathrm{~s}$ & \\
\hline cavdet 1 & 70 & 4 & $1.275 \mathrm{~s}$ & \\
\hline
\end{tabular}

\begin{tabular}{|l|c|l|l|l|}
\hline benchmark & $\mathrm{LOC}$ & $d_{l}$ & time & Feaut. \\
\hline cavdet 2 & 96 & 4 & $0.820 \mathrm{~s}$ & \\
\hline cavdet 3 & 93 & 4 & $0.762 \mathrm{~s}$ & \\
\hline cavdet 4 & 53 & 4 & $0.425 \mathrm{~s}$ & \\
\hline cavdet 5 & 53 & 4 & $0.408 \mathrm{~s}$ & \\
\hline cavdet 6 & 54 & 4 & $0.388 \mathrm{~s}$ & \\
\hline cavdet 7 & 54 & 4 & $0.388 \mathrm{~s}$ & \\
\hline cavdet 8 & 53 & 4 & $0.310 \mathrm{~s}$ & \\
\hline cavdet 9 & 61 & 4 & $0.651 \mathrm{~s}$ & \\
\hline
\end{tabular}

Table 1. Benchmarks with transformation times.

\section{Experimental results}

We have implemented our DSA transformation using the Omega library [15] for modeling and simplifying the iteration domains and for finding the extremal values of the iterators. Our implementation has full support for data dependent indexation and conditions, and partial support for extra data types from $\mathrm{C}$ (e.g., structs).

We have applied the transformation to a number of multimedia kernels: Gaussian elimination without pivoting (gauss1 and gauss2) and with pivoting (gauss_dd1 and gauss_dd2), a cavity detector (cavdet 1 through cavdet 9 ), a MP3 decoder ( $m p 3 \_1$ and $\left.m p 3 \_2\right)$ and qsdpcm motion estimation (qsdpcm). These benchmarks are shown in Table 1. The LOC column lists the number of lines of code, and the $d_{l}$ column lists the maximum loop nest depth. The DSA transformation was run on a Pentium $42.4 \mathrm{GHz}$ with $768 \mathrm{MB}$ RAM.

Of these benchmarks, only gauss1 and gauss2 do not contain data dependent indexation or conditions and can be handled by [7]. For example gauss_dd1 and gauss_dd2 search for a pivot and then swap rows depending on the location of the pivot. This location depends on the original matrix elements in a non-trivial way and changes with each iteration. This makes that these programs cannot easily be transformed to a form that [7] would be able to handle. The benchmarks that can be handled by [7] are marked in the last column of Table 1 .

For each benchmark (except qsdpcm) the first version is the original version of the code. The other versions are obtained by applying transformations in order to increase locality of access as well as regularity of access usually by aligning loops and merging them. The effect is that uses and definitions of array elements are moved closer to each other. Because life times of array elements become much smaller, fewer elements are live at each point and less memory is needed to store them. An effect noticeable from Table 1 is that DSA transformation times tend to go down with the amount of locality/regularity improving transformations applied. This is because when the reaching definitions are close to the uses, they are easier to find. Additionally, fewer reaching definitions need to be discerned and the conditions governing them become smaller. The bene- 


\begin{tabular}{|c|c|c|c|c|c|}
\hline$n$ & 1 & 2 & 10 & 25 & 50 \\
\hline$t$ & $1.362 \mathrm{~s}$ & $2.679 \mathrm{~s}$ & $13.20 \mathrm{~s}$ & $32.93 \mathrm{~s}$ & $65.95 \mathrm{~s}$ \\
\hline$t / n$ & $1.362 \mathrm{~s}$ & $1.339 \mathrm{~s}$ & $1.320 \mathrm{~s}$ & $1.317 \mathrm{~s}$ & 1.319 \\
\hline
\end{tabular}

Table 2. Scalability experiment.

ficial effect of regularity and locality can even outweigh an increase in program size, as illustrated by cavdet 1 vs cavdet 2 . Note that the time for cavdet 9 is longer than for cavdet8, which seems to go against the general trend. But in cavdet 9 heavily used array elements are copied into smaller arrays, which can be placed in a smaller memory that is faster and consumes less energy [5]. Besides making the program larger, this makes the data flow more complex again. Intuitively, the reason is that these arrays are added when there are many uses for a single definition, and this single definition usually cannot be put close to each use.

To ascertain that our method scales well with growing program size, we have run our DSA transformation on a program of growing size. Usually two programs of differing size have a different loop structure as well, even if the maximum loop depth is the same. This causes a lot of noise on the measurements. A very clear example is the difference between gauss 1 and $q s d p c m$ : an increase of roughly a factor 30 in LOC, but an increase of 1750 in transformation time. At play here is the great increase in loop depth. To reduce this noise we have opted to chain the cavity detector kernel (an adaptation of cavdet1) together a number of times (denoted $n$ ) with the output array of one instance of the cavity detector the same as the input array of the next instance. This way we can build reasonable programs of a size that is a multiple of the original cavity detector, but with the extra that the code that is added each time has a comparable loop structure. The measurements are shown in Table 2. The transformation time is shown as $t$, and the transformation time per instance of the cavity detector is shown as the ratio $t / n$. This ratio actually decreases, but this is due to a constant startup cost of which each instance's share decreases as the number of instances increases. Another measure for the transformation time for one instance of the cavity detector could be the difference in value for $t$ for $n=1$ and $n=2$. This difference is 1.317 which $t / n$ seems to converge to, increasing our confidence that our method indeed behaves linearly.

For comparison with the DSA transformation of [7], we have done some experiments with $\mathrm{f} 2$ sare [2], an implementation of [7]. This tool broke down for $n=3$ in the scalability experiment as it did not give an answer after several hours of computation. Note though that $\mathrm{f} 2$ sare is hardly a robust tool, and other, more robust, implementations exist but they (e.g., MatParser [10]) are usually part of a larger system, so accurate measurements of the DSA transformation only are difficult. Moreover, MatParser [10] is not publicly available. 


\section{Conclusion}

In this paper we have presented a new method that transforms programs to dynamic single assignment which is in practice linear in the size of the program (for a constant loop depth). This is achieved by adding copy operations in such a way that we can use an exact scalar reaching definitions analysis and a simple way of determining the indexation for each of these reaching definitions whereas existing methods need an expensive, exact array data flow analysis. We have implemented this DSA transformation and it is currently being used as an enabling step for functional verification [20,23] and memory optimizations [5]. The extra copy operations can be removed by advanced copy propagation [24]. We plan to investigate the interaction between our new DSA transformation and copy propagation as part of future work. On the one hand experiments show that for non-data-dependent code (e.g., gauss 1 and gauss 2 from Fig. 1) all copy operations can be removed, and the resulting code is equivalent to the code Feautrier's method returns. There is the option though to leave some of the copy operations. This would allow to control the complexity of the transformation as well as the complexity of the resulting program as both are tightly linked. This complexity is due to the number of cases that need to be discerned, often due to border conditions which give rise to few copy operation instances that are not worth removing given the cost of doing so. The investigation of this trade-off of the remaining overhead versus transformation time and code complexity. On the other hand, for programs involving data-dependent indexation or conditions, not all copy operations can be removed as that would imply that the program is fully analyzable which is not so in general. In future work, we want to determine how far copy propagation can go in general.

\section{References}

1. A. V. Aho, R. Sethi, and J. D. Ullman. Compilers: Principles, Techniques and Tools. Addison-Wesley, Inc., 1986.

2. C. Alias. f2sare. http://www.prism.uvsq.fr/users/ca/progs/f2sare.tgz, 2003.

3. R. A. Ballance, A. B. Maccabe, and K. J. Ottenstein. The program dependence web: A representation supporting control-, and demand-driven interpretation of imperative languages. In Proceedings of PLDI'90, pages 257-271, 1990.

4. J. Bu and E. Deprettere. Converting sequential interative algorithms to recurrent equations for automatic design of systolic arrays. In Proceedings of ICASSP '88, volume vol IV, pages 2025-2028. IEEE Press, 1988.

5. F. Catthoor, S. Wuytack, E. De Greef, F. Balasa, L. Nachtergaele, and A. Vandecappelle. Custom Memory Management Methodology: Exploration of Memory Organisation for Embedded Multimedia System Design. Kluwer Academic Publishers, 1998.

6. R. Cytron, J. Ferrante, B. K. Rosen, M. N. Wegman, and F. K. Zadeck. Efficiently computing static single assignment form and the control dependence graph. ACM Transactions on Programming Languages and Systems, 13(4):451-490, October 1991. 
7. P. Feautrier. Array expansion. In Proceedings of the Second International Conference on Supercomputing, pages 429-441, St. Malo, France, 1988.

8. D. Genin and P. Hilfinger. Silage Reference Manual, Draft 1.0. Silvar-Lisco, Leuven, 1989.

9. R. Karp, R. Miller, and S. Winograd. The organization of computations for uniform recurrence equations. In Journal of the ACM, volume 14, pages 563-590, July 1967.

10. B. Kienhuis. Matparser: An array dataflow analysis compiler. Technical report, University of California, Berkeley, February 2000.

11. B. Kienhuis, E. Rijpkema, and E. Deprettere. Compaan: Deriving process networks from matlab for embedded signal processing architectures. In 8th International Workshop on Hardware/Software Codesign, pages 13-17, 2000.

12. K. Knobe and V. Sarkar. Array SSA form and its use in parallelization. In Symposium on Principles of Programming Languages, pages 107-120, 1998.

13. Z. Li. Array privatization for parallel execution of loops. In ICS '92: Proceedings of the 6th international conference on Supercomputing, pages 313-322, New York, NY, USA, 1992. ACM Press.

14. C. Offner and K. Knobe. Weak dynamic single assignment form. Technical Report HPL-2003-169, HP Labs, Aug 2003.

15. W. Pugh. The Omega test: A fast and practical integer programming algorithm for dependence analysis. In Proceedings of Supercomputing '91, Albuquerque, NM, 1991.

16. F. Quilleré and S. Rajopadhye. Optimizing memory usage in the polyhedral model. ACM Transactions on Programming Languages, 22(5):773-815, 2000.

17. P. Quinton. Automatic synthesis of systolic arrays from uniform recurrent equations. In ISCA '84: Proceedings of the 11th annual international symposium on Computer architecture, pages 208-214, New York, NY, USA, 1984. ACM Press.

18. S.-B. Scholz. Single Assignment C - Functional Programming Using Imperative style. In Proceedings of the 6th International Workshop on Implementation of Functional Languages (IFL'94), pages 21.1-21.13, 1994.

19. A. Schrijver. Theory of Integer and Linear Programming. John Wiley and Sons, 1986.

20. K. Shashidhar, M. Bruynooghe, F. Catthoor, and G. Janssens. An automatic verification technique for loop and data reuse transformations based on geometric modeling of programs. Journal of Universal Computer Science, 9(3):248-269, 2003.

21. K. Shashidhar, M. Bruynooghe, F. Catthoor, and G. Janssens. Automatic verification of source code transformations on array-intensive programs: demonstration with real-life examples. Technical Report CW 401, Department of Computer Science, Katholieke Universiteit Leuven, Belgium, 2005.

22. K. Shashidhar, M. Bruynooghe, F. Catthoor, and G. Janssens. Functional equivalence checking for verification of algebraic transformations on array-intensive source code. In Proceedings of Design, Automation and Test in Europe, Munich, Germany. IEEE Computer Society, 2005.

23. K. Shashidhar, M. Bruynooghe, F. Catthoor, and G. Janssens. Verification of source code transformations by program equivalence checking. In Proceedings of 14th International Conference on Compiler Construction, Edinburgh, Scotland, 2005.

24. P. Vanbroekhoven, G. Janssens, M. Bruynooghe, H. Corporaal, and F. Catthoor. Advanced copy propagation for arrays. In Languages, Compilers, and Tools for Embedded Systems LCTES'03, pages 24-33, June 2003. 